\title{
The Mystery of Missing Species in Atom Probe Tomography of Composite Materials
}

\author{
M. Karahka ${ }^{1}$ and H. J. Kreuzer ${ }^{1}$ \\ ${ }^{1}$ Department of Physics and Atmospheric Science, Dalhousie University, Halifax, Nova Scotia B3H \\ 3J5, Canada
}

Although originally developed for metallic tips and used extensively for the study of the atomic composition of steels and other alloys atom probe tomography has also been adapted for the study of semiconductors and more recently for insulators through the assistance of ultrafast laser pulses. A number of outstanding issues have recently been resolved for APT of insulators and semiconductors including the discovery that at high fields the bandgap in semiconductors and insulators goes to zero leading to their metallization [1,2]. A very interesting puzzle, however, remains unsolved, namely that even in stoichiometric samples of oxides, as an example, one observes non-stoichiometric ion yields, namely that there are missing oxygen ions depending on the field strength and on the oxide itself with e.g. different behavior for $\mathrm{MgO}$ and $\mathrm{ZnO}$ as examples. Similarly, for $\mathrm{AlN}$ and GaN the nitrogen yields are always less, and in alkine halides there are less halide ions [3,4]. This is a serious problem as it precludes a proper compositional analysis of oxides and other insulators and semiconductors by APT. In a recent Letter we presented a quantitative model that explains the non-stoichiometry allowing a fit to experimental data of ion yields as a function of applied field to extract activation barriers and prefactors [5]. The numbers are confirmed by density functional theory. We also show that for oxides the missing oxygen is thermally desorbed as neutral O2, either directly or associatively. Finally, we suggested methods to improve the experimental setup.

The model is based on the Arrhenius parametrization of the ion yield with the activation energy given by a quadratic dependence on the applied field. The ratio of metal to oxygen yield $\mathrm{R}$ as a function of normalized field $\mathrm{f}$ is shown in Fig. 1 (a). To understand the physics behind the non-stoichiometry of ion yields in APT we need to look at the binding energies and evaporation fields of the oxides obtained from DFT calculations of a $\mathrm{Zn}_{26} \mathrm{O}_{26}$ wurtzite cluster. Obviously corner atoms are least bound, e.g. a corner $\mathrm{Zn}$ is bound by $5.2 \mathrm{eV}$ and a corner $\mathrm{O}$ by $7.8 \mathrm{eV}$ whereas those atoms in the interior of a surface plane are more strongly bound by an additional $2 \mathrm{eV}$. As a result the evaporation field of the corner $\mathrm{Zn}$ and $\mathrm{O}$ is $2.6 \mathrm{~V} / \AA$ and $3.2 \mathrm{~V} / \AA$, respectively. Thus as the field is increased $\mathrm{Zn}$ is removed first as $\mathrm{Zn}^{2+}$ leaving behind an oxygen-rich cluster $\mathrm{Zn}_{26} \mathrm{O}_{25}$ from which further $\mathrm{Zn}$ ions evaporate instead of oxygen. A similar scenario is in place for $\mathrm{MgO}$. One is tempted to suggest that a way to avoid O-enrichment during field evaporation is to crank up the field to the strength of $\mathrm{O}$ evaporation. This however, is impractical as the cluster "melts" i.e. its crystalline structure is destroyed so that APT will not provide any information on the crystallographic structure of the specimen at all.

Because APT yields non-stoichiometric ion yields from oxides we need to find a second channel by which $\mathrm{O}$ is removed. There are two options: negative $\mathrm{O}$ ions can migrate down the surface of the tip and eventually desorb thermally after forming $\mathrm{O}_{2}$. With atomic $\mathrm{O}$ accumulating at the tip, we have, in addition to surface diffusion, two possible desorption channels for its removal: (i) molecular oxygen forms on the surface; this seems to be the case for $\mathrm{ZnO}$ [6], or (ii) associative desorption, i.e. $2 \mathrm{O}_{\mathrm{ad}} \rightarrow \mathrm{O}_{2}{ }^{\text {(gas) }}$ where two adsorbed $\mathrm{O}$ atoms need their surface binding energy $\mathrm{V}_{\mathrm{O}}(\mathrm{F})$ to break their surface bonds and gain the molecular binding energy or dissociation energy $\mathrm{D}_{\mathrm{O}_{2}}$ in the gas phase. 
Trying to demystify the issue of missing oxygen in APT ion yields of oxides we have shown: (1) Metal atoms in an oxide are typically less bound than $\mathrm{O}$ and thus can be field evaporated at lower fields. (2) Although some $\mathrm{O}$ can be field evaporated e.g. as $\mathrm{O}_{2}{ }^{+}$the surface will be enriched with $\mathrm{O}$ which however, is not bound too strongly at high fields and can easily migrate over the surface. Thus thermal desorption of preexisting neutral molecules or associatively from atomic $\mathrm{O}$ will remove the excess $\mathrm{O}$. However, these species, being neutral, will not be detected as ions. (3) Increasing the field above the evaporation field strength of the metal component is usually not an option to obtain stoichiometry as the higher fields will "melt" the surface structure invalidating the aim that APT can obtain site-specific atomic maps layer-by-layer.

The question then remains on how to make APT work stoichiometrically for oxides. We have several suggestions: (1) For some, but not all oxides there seems to be an optimal field where stoichiometry can be maintained, e.g. the maximum field for the $\mathrm{ZnO}$ system depicted in Fig. 1 (b), or the cross-over field in Fig. 1 (a). (2) Block's pulse mode will clean the surface during the high field pulsed which would come off as ions although no longer site specific. (3) As our results in this paper and in previous publications have made amply clear DFT calculations can provide guidelines on optimal fields and also on the binding characteristics that ultimately dictate which ions come off and which desorb thermally as neutrals. Ideally a comprehensive catalogue of relevant atomic data would be generated and made available. These remedial methods may however distort the surface themselves so some serious testing is required. As a final statement we would like to repeat the obvious: Although we have dealt exclusively with oxides in this paper our approach also explains the absence of stoichiometry in nitrides (GaN, AlN etc.) and in alkali halides.
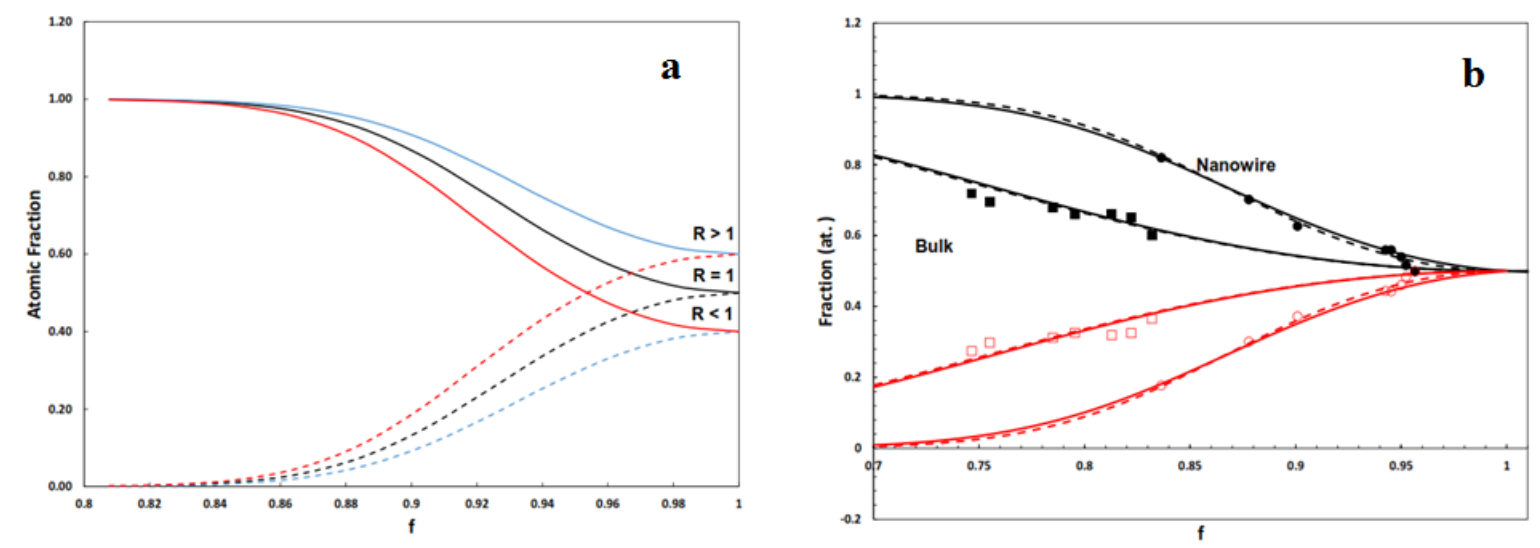

Figure 1. (a) Schematic of ion fractions, upper curves (solid lines) for metal ions and lower curves (dashed lines) for oxygen ions, for different values of $\mathrm{R}$. (b) Ion fractions of $\mathrm{Zn}^{+}$and $\mathrm{O}^{+}$from a $\mathrm{ZnO}$ tip. Dashed lines from fitting the model to the experimental data; solid curve calculated with DFT parameters. The experimental data points were taken from [3].

[1] E. P. Silaeva, M. Karahka, and H.J. Kreuzer, Curr. Opin. Solid State Mater. Sci. 17 (2013), p. 211. [2] E.P. Silaeva et al, Nano Letters 14 (2014), p. 6066.

[3] L. Mancini et al, J. Phys. Chem. C 118 (41) (2014), p. 24136.

[4] A. Devaraj, private communication.

[5] M. Karahka, Y. Xia, and H. J. Kreuzer, Applied Physics Letters 107 (2015), p. 062105.

[6] M. L. Karahka, and H.J. Kreuzer, Ultramicroscopy 159 (2015), p.156.

[7] This work was supported by grants from the Natural Sciences and Engineering Council of Canada and from the Office of Naval Research, Washington DC. We would also like to thank Arun Devaraj and Lorenzo Rigutti for sharing some of their experimental results before publication. 